\title{
Sexe, Type De Familiarite Et Perception Du Handicap Mental Chez Des Individus Vivant En Cote d'Ivoire (Cas De La Trisomie 21)
}

\author{
Dr. Sahi Banty Isabelle \\ Dr. Tra bi Tra Isidore \\ Dr. Sahi Koffi Dieudonné Ibrahim \\ Département de Psychologie, \\ Université Félix Houphouët-Boigny d’Abidjan, Côte d'Ivoire
}

Doi:10.19044/esj.2019.v15n16p45 ～URL:http://dx.doi.org/10.19044/esj.2019.v15n16p45

\section{Résumé}

La présente étude vise à examiner l'influence du sexe et du type de familiarité des individus sur leur perception du handicap mental, notamment celle de la trisomie 21. Elle porte sur un échantillon composé de 100 sujets dont certains sont en formation à l'Institut Nationale de Formation Sociale (INFS) et d'autres sont des individus appartenant à l'environnement immédiat des enfants porteurs de trisomie 21. Ces groupes de sujets obtenus à l'aide de la technique d'échantillonnage par choix raisonné comporte 50 hommes et 50 femmes dont l'âge est compris entre 21 et 54 ans. Un questionnaire d'identification a permis de déterminer le sexe et le type de familiarité des sujets. La perception de la trisomie 21 est, quant à elle, évaluée par un questionnaire élaboré en se référant à celui de Hetebrij et Racine (2011). Les résultats obtenus indiquent que l'influence de type de familiarité sur la perception de la trisomie 21 est significative, alors que celle du sexe ne l'est pas. De tels résultats suggèrent de considérer ces facteurs dans la prise en charge des personnes handicapées en général et celles porteuses de la trisomie 21 en particulier.

Mots-clés: Sexe, type de familiarité, Perception, Handicap mental, trisomie 21 


\title{
Gender, Type of Familiarity and Perception of Mental Disability in Individuals Living in Cote d'Ivoire (Case Of Trisomy 21)
}

\author{
Dr. Sahi Banty Isabelle \\ Dr. Tra bi Tra Isidore \\ Dr. Sahi Koffi Dieudonné Ibrahim
}

Département de Psychologie, Université Félix Houphouët-Boigny d’Abidjan, Côte d'Ivoire

\begin{abstract}
This paper focuses on examining the influence of gender and the type of familiarity of individuals on their perception of mental disability, particularly that of trisomy 21. A sample of 100 subjects was used for the study, some of whom are undergoing training at the Institute. National Social Training Institute (NSTI) and others are individuals belonging to the immediate environment of children with trisomy 21. These groups of subjects were obtained using the reasoned choice sampling technique which comprises of 50 men and 50 women between 21 and 54 years old. An identification questionnaire was used to determine the sex and type of familiarity of the subjects. The perception of Trisomy 21 is, in turn, assessed by a questionnaire developed with reference to that of Hetebrij and Racine (2011). The results obtained indicate that the influence of type of familiarity on the perception of trisomy 21 is significant, while that of sex is not. Such results suggest considering these factors in the care of people with disabilities in general and those with trisomy 21 in particular.
\end{abstract}

Keywords: Gender, type of familiarity, perception, mental disability, down syndrome

\section{Introduction}

Le souhait de tout individu est de constater une évolution saine de sa progéniture. Mais force est de constater que tous les enfants ne sont pas logés à la même enseigne dans la mesure où certains sont porteurs de handicaps.Le rapport mondial sur le handicap publié par l'Organisation Mondiale de la Santé (OMS) en 2001 révèle que plus de 120 millions de personnes sont concernées par le handicap mental dans le monde. Parmi les handicaps mentaux, le Syndrome de Down ou trisomie 21est l'aberration 
chromosomique la plus fréquente et reste la cause première du retard mental (OMS, 2008).

Etant donné que les personnes porteuses de trisomie ont un profil cognitif particulier, elles sont l'objet de stéréotypes, de croyances irrationnelles tant dans les structures éducatives que les organisations (Hetebrij \& Racine, 2011). Ces personnes sont stigmatisées, voire marginalisées. Cette situation est très préoccupante en Côte d'Ivoire car ce phénomène semble prendre de l'ampleur. En effet, les enfants porteurs de handicaps mentaux sont le plus souvent suspectés d'être des "enfantsserpents", des sorciers ou des "enfants porte-malheurs" dans la conception populaire traditionnelle, Ainsi, des rites d'accompagnement, par l'ordre ancestral, sont prévus pour les supprimer. Ceux qui vivent en milieu urbain sont laissés à la charge de substituts parentaux, notamment les grands-parents (Attrobra, 2015).

Face aux conduites discriminatives dont souffrent les nombreuses personnes en situation de handicap mental et leurs familles, l'État de Côte d'ivoire ainsi que des Organisations non Gouvernementales (ONG) nationaleset internationales développent des actions et exécutent divers programmes en leur faveur pour atténuer les souffrances de cette frange de la population. Toutefois, nonobstant toutes ces initiatives, la perception que les individus ont des personnes porteuses de trisomie 21 n'a pas fondamentalement changé. Il y a donc lieu de s'interroger sur les facteurs susceptibles d'expliquer les perceptions des individus à l'égard des personnes trisomiques.

Des travaux ont été effectués dans cette perspective. Ainsi et par exemple, Bruner et Goodman (1947), Duru-Bellat (1994,1995), Djider, Murat et Robin (2003), et Benacer (2005) distinguent deux grands types de facteurs influençant la perception. Ce sont entre autres les déterminants structuraux qualifiés d'innés et peu modifiables (facteurs psychobiologiques) et lesvariables de milieu (expériences antérieures, croyances). Parmi ces facteurs, le sexe de l'individu et le type de familiarité qu'il entretient avec la personne porteuse de trisomie 21 pourraient, nous semble-t-il, influer sur sa perception de cette dernière. Toutefois, en l'état actuel de nos connaissances, il apparaît que les travaux menés dans ce domaine n'ont pas pris en compte ces variablesen tant que facteurs susceptibles d'influencer la perception du handicap mental. C'est pourquoi, nous envisageons de mener une étude, visant à comparer les perceptions de femmes et d'hommes entretenant une familiarité soit cognitive, soit relationnelle avec des enfants porteurs de trisomie 21 .

Une telle étude pourrait, d'un point de vue théorique, permettre de comprendre l'émergence des conduites discriminatives à l'égard des personnes handicapées mentales. Au plan pratique elle pourraitcontribuerà une prise en charge efficace des personnes en situation de handicap mental. 
Elle pourrait également susciterla sensibilisation des populations à leur intégration aussi bienen milieu scolaire que professionnel. En définitive, nous voulons à travers la présente étude, montrerque la perception du handicap mental chez les individus varie en fonction de leur sexe et dutype de familiarité qu'ils ont avec les handicapés.

$\mathrm{Au}$ regard de cet objectif et des idées qui découlent des travaux examinés, nous conjecturons que la proportion des individus entretenant une familiarité de type relationnel avec les personnes trisomiques et qui ont une perception positive de ces dernières est supérieure à celle de leurs homologues qui entretiennent une familiarité de type cognitif avec celles-ci. En outre, nous postulons que le nombre de femmes qui ont une perception positive des personnes trisomiques est supérieur à celui des hommes. Ces deux hypothèses sont mises à l'épreuve des faits à l'aide de la démarche méthodologique qui suit.

\section{1- Méthode}

La partie consacrée à la méthode porte successivement sur les variables de l'étude, l'échantillon, les instruments de mesure et la procédure de collecte des données.

\section{1-1- Variables de l'étude 1-1-1- Variable indépendante a- Sexe}

Selon Le Maner-Idrissi (1997), le sexe renvoie aux différences entre mâles et femelles. Autrement dit, la notion de sexe fait référence aux caractéristiques biologiques, psychologiques et socioculturelles permettant de distinguer les hommes des femmes. C'est donc une variable qualitative dichotomique (homme, femme). Les hommes produisent plus de testostérone, source d'une plus grande virilité, agressivité et antipathie (Ayral, 2011; BaronCohen, 2003; Boudieu, 1998). Par ailleurs, dans l'éducation de ceux-ci, les expressions motrices que l'on associe au dynamisme et à l'indépendance sont valorisées (Kanga, 2018; N'douba, 2011; Vouillot, 2002). De ce fait, les hommes sembleraient caractérisés par la froideur, l'objectivité et l'esprit de domination. A l'opposée chez les femmes, les hormones qui ont le taux le plus élevé sont l'œstrogène et la progestérone (LLedo \& Vinvent, 2012; Solano, 2010). Elles sont à l'origine de la tranquillité, de la sympathie et d'une plus grande sensibilité sociale manifestées par ces dernières (Eagly \& Crowley, 1986). De plus, le processus de socialisation auquel elles sont soumises tendrait à développer, chez elles, la patience, l'amour du prochain et l'assistance des autres (Fournier, 2014).

Il apparaît donc que les différences biologiques et physiologiques entre hommes et femmes, ainsi que celles dans la façon dont ils sont éduqués dans 
le contexte ivoirien, pourraient engendrer des variations dans leur manière de percevoir les enfants trisomiques 21 .

\section{b- Type de familiarité}

La familiarité réfère au nombre d'expériences liées à un objet ou à une situation accumulée par un individu. En d'autres termes, elle revoie aux expositions à cette situation, ainsi que la recherche d'informations sur celle-ci (Rohmer \& Louvet, 2000). Elle peut se diversifier selon le mode d'appréhension de la situation ou le type de relation établi avec autrui. Ainsi, on pourrait distinguer la familiarité cognitive de la familiarité relationnelle.

Les individus ayant le type de familiarité cognitif se caractérisent par leurs connaissances de la déficience. Ils ont une familiarité avec la déficience en termes de savoirs. En effet, ces individus n'entretiennent aucun lien ou contact physique avec les personnes trisomiques 21 . Elles ont simplement une connaissance théorique du handicap mental et notamment celle de la trisomie 21. Par contre, les individus qui entretiennent la familiarité de type relationnel sont ceux qui côtoient quotidiennement les personnes porteuses de trisomie 21. Ces individus proches des trisomiques pourraient les identifier comme des semblables présentant quelques difficultés remédiables (Dovidio, Gaertner \& Loux, 2000).

En somme, le type de familiarité est une variable qualitative ayant deux modalités (relationnel, cognitif) qui pourraient, par leur manifestation, influer différemment sur la façon de percevoir les personnes trisomiques 21 .

\section{1-1-2- Variable dépendante}

La variable à mesurer dans la cet article correspond à la perception de la trisomie 21 chez des individus. La perception est le processus par lequel l'individu recueille et traite l'information en vue de s'adapter à son milieu de vie (Reuchlin, 1996; Shachter \& Singer, 1962). C'est par conséquent une fonction cognitive indispensable à sa survie. Les situations particulières telles que le handicap mental et singulièrement la trisomie 21 peuvent être l'objet de perceptions diverses. Ainsi, l'individu peut avoir une perception plus ou moins positive de la personne trisomique. Et, cette perception peut être appréhendée à travers les opinions, les attitudes comportementales et le degré d'empathie exprimés à l'endroit de cette personne physiquement difforme et mentalement déficiente. Compte tenu du nombre important de sujets (100) à examiner, l'accent est ici mis sur l'opinion parce que celle-ci récapitule ou permet de saisir la perception de l'individu.

\section{1-2- Echantillon de l'étude}

La technique d'échantillonnage par choix raisonné nous a permis de sélectionner 100 sujets (hommes et femmes entretenant une familiarité de type 
cognitif ou relationnel avec les enfants trisomiques) tout en contrôlant les variables parasites susceptibles de faire varier la perception à l'égard de la trisomie 21 (âge, niveau d'instruction, la profession, le niveau socioéconomique). Cet échantillon est composé de 50 hommes et 50 femmes provenant de l'Institut Nationale de Formation Social (INFS) et du milieu familial des enfants porteurs de trisomie 21. Les étudiants de l'INFS entretiennent le type de familiarité cognitif avec les enfants trisomiques. Par contre, les parents de ces enfants ou leurs substituts entretiennent une familiarité de type relationnelavec ces derniers. L'échantillon ainsi constitué est composé de quatre groupes équivalents en ce qui concerne les critères de sélection et contrastés au plan du sexe et du type de familiarité, comme l'indique le Tableau I.

Tableau I. Groupes expérimentaux

\begin{tabular}{llll}
\hline Sexe & $\begin{array}{l}\text { Familiarité de } \\
\text { type cognitif }\end{array}$ & $\begin{array}{l}\text { Familiarité de } \\
\text { type } \\
\text { relationnel }\end{array}$ & Total \\
\hline Homme & $25(\mathrm{G} 1)$ & $25(\mathrm{G} 2)$ & $\mathbf{5 0}$ \\
Femme & $25(\mathrm{G} 3)$ & $25(\mathrm{G} 4)$ & $\mathbf{5 0}$ \\
Total & $\mathbf{5 0}$ & $\mathbf{5 0}$ & $\mathbf{1 0 0}$ \\
\hline
\end{tabular}

C'est la perception de ces participants au sujet de la trisomie 21 que nous avons mesurée.

\section{1-3- Instruments de mesure}

Notre étude comporte trois variables dont deux variables indépendantes (sexe et type de familiarité) et une variable dépendante (perception des enfants porteurs de trisomie 21). Les instruments de mesure réfèrent aux outils utilisés pour les appréhender. Les données de l'étude ont été essentiellement recueillies à l'aide de trois instruments que sont le questionnaire d'identification, le questionnaire de Hetebrij et Racine (2011) que nous avons adapté aux caractéristiques des participants à l'étude et un guide d'entretient semi- directif. Outre les items relatifs aux caractéristiques des participants (sexe, type de familiarité, niveau d'études, profession, niveau socioéconomique...), ce questionnaire comporte 21 items sur une échelle de type Lickert en cinq (5) points: (1) « je ne suis pas tout à fait d'accord »; ((2) «je ne suis pas d'accord »; (3) « je ne sais pas»; (4) « je suis d'accord»; (5) « je suis tout à fait d'accord ». 


\section{1-4- Procédure de collecte des données}

Dans un premier temps, nous avons utilisé un questionnaire d'identification pour sélectionner les participants et contrôler les variables parasites. En second lieu, nous avons eu recours à un questionnaire adapté relativement à celui de Hetebrij et Racine (2011) pour évaluer leur perception à l'égard des personnes trisomiques. Dans troisième et dernier temps, nous avons mené des entretiens semi-dirigés avec les participants enfin de mieux appréhender leur perception des enfants porteurs de trisomie 21. Eu égard à la nature qualitative des variables à l'étude, le Khi Carré de Pearson a été utilisé comme technique pour le traitement statistique des données collectées. Par ailleurs, une analyse de contenu du discours des participants est réalisée. Et, les résultats obtenus à l'issue de ce traitement sont présentés et analysés dans la section qui suit.

\section{2- Résultats}

Les résultats sont présentés sous forme de tableaux et suivant les hypothèses émises. Le tableau II examine les perceptions que les individus ont des enfants porteurs de trisomie 21 selon qu'ils entretiennent une familiarité de type cognitif ou relationnel avec ces derniers.

Tableau II. Répartition des perceptions des sujets en fonction du type de familiarité

\begin{tabular}{llll}
\hline Type de familiarité & $\begin{array}{l}\text { Perception } \\
\text { négative }\end{array}$ & $\begin{array}{l}\text { Perception } \\
\text { positive }\end{array}$ & Total \\
\hline Cognitif & 34 & 16 & $\mathbf{5 0}$ \\
Relationnel & 16 & 34 & $\mathbf{5 0}$ \\
Total & $\mathbf{5 0}$ & $\mathbf{5 0}$ & $\mathbf{1 0 0}$ \\
\hline \multicolumn{5}{r}{$=12,96$ (Significatif, $\mathrm{p}=.05)$}
\end{tabular}

L'utilisation du khi carré dans le cas de la comparaison d'échantillons indépendants montre qu'il y a une différencesignificative entre les deux groupes d'individus $\left(\chi^{2}=12,96\right.$ à $\left.1 \mathrm{ddl}, \mathrm{P}=.05\right)$. L'analyse minutieuse du Tableau II laisse transparaître que les individus qui entretiennent une familiarité de type relationnel avec les personnes trisomiques sont plus nombreux à avoir une perception positivede ces dernières que leurs homologues entretenant une familiarité de type cognitif avec celles-ci. Le contenu du discours des participants va également dans ce sens. Qu'en est-il de la relation entre le sexe de l'individu et sa perception de la trisomie 21. Le Tableau III indique les proportions d'hommes et de femmes selon que ceuxci ou celles-ci ont une perception positive ou négative de la trisomie 21 . 
Tableau III. Répartition des perceptions des sujets en fonction du sexe

\begin{tabular}{llll}
\hline Sexe & $\begin{array}{l}\text { Perception } \\
\text { négative }\end{array}$ & $\begin{array}{l}\text { Perception } \\
\text { positive }\end{array}$ & Total \\
\hline Homme & 23 & 27 & $\mathbf{5 0}$ \\
Femme & 27 & 23 & $\mathbf{5 0}$ \\
Total & $\mathbf{5 0}$ & $\mathbf{5 0}$ & $\mathbf{1 0 0}$ \\
\hline & $\chi^{2}=0,64$ (Non significatif)
\end{tabular}

Le Tableau III ci-dessus nous révèle qu'il n'existe pas de différence significative entre les groupes comparés $\left(\chi^{2}=0,64\right.$ à $\left.1 \mathrm{ddl}, \mathrm{P}=.05\right)$ en ce qui concerne la perceptionqu'ils ont des personnes porteuses de trisomie 21. Par conséquent, les hommes présentent une perception sensiblement équivalente à celle des femmes au sujet de ces personnes. Ces résultats que nous avons obtenus sont expliqués à la lumière des théories du contact intergroupede Allport (1954), de « l'apparaisal » d'Arnold (1960), de la socialisation sexuée de Maccoby (1974) et confrontés à ceux d'auteurs ayant antérieurement exploré le domaine que nous étudions dans la partie consacrée à la discussion des résultats.

\section{3- Discussion}

Nous avons supposé que le type de familiarité et le sexe des individus influencent leur perception des enfants porteurs de trisomie 21. A l'issue de nos investigations sur le terrain, nous avons abouti à des résultats plus ou moins mitigés. Le premier résultat confirme que les individus qui entretiennent une familiarité de type relationnel (interaction directe) avec les personnes trisomiques sont plus nombreux à avoir une perception positive de ces dernières que leurs homologues qui entretiennent une familiarité de type cognitif (sans interaction directe) avec celles-ci. Par contre, le second résultat montre qu'il n'y a pas de lien entre le sexe de l'individu et sa perception des enfants porteurs de trisomie 21. En effet, l'hypothèse postulant quele nombre de femmes ayant une perception positive des personnes trisomiques est supérieur à celui de leurs pairs de sexe masculin est infirmée par les faits.

En ce qui concerne le premier résultat obtenu, il concorde avec ceux d'études antérieures (Zajonc, 1997, 2000; Cross, Halcomb \& Matter, 1967; Langer, Bashner \& Chanowlzt, 1985; Rohmer \& Louvet, 2000; Dovidio, Gaertner \& Loux, 2000; Bless, 2009), qui supposent que les individus ayant un contact avec des personnes handicapées ont une attitude plus positive à leurs égards que leurs pairs qui n'entretiennent pas de relation avec ces dernières. Autrement dit, plus on a de contacts avec des personnes déficientes, plus nos attitudes envers elles deviennent positives. Cela pourrait s'expliquer parl'effet de la familiarité rendue possible par la fréquence des contacts. De 
fait, le contact participerait àune diminution maximale de préjugés et des réactions émotionnelles négatives. C'est probablement ce qui permet aux individus qui entretiennent le type de familiarité relationneld'avoir une perception positive de la trisomie 21. Cette perception est contraire à celle de leurs homologues qui entretiennent une familiarité de type cognitif avec ces dernières. Ainsi, le type de familiarité relationnel peut être considéré comme un facteur susceptibled'améliorer la façon de percevoir une catégorie d'individus. Dans la mesure où le contact peut rendre la perception que l'on a d'autrui plus positive qu'auparavant, il apparait clairement que les participants familiarisés avec les personnes handicapées sont celles qui peuvent interagir aisément avec elles. Ceci implique plus d'immersion des étudiants en formation afin qu'ils puissent véritablement être imprégnés des réalités des individus qu'ils auront probablement la charge dans leur carrière professionnelle.

Pour ce qui a trait au second résultatrelatif à l'influence du sexe sur la perception à l'égard des enfants trisomiques, il reflète la vive controverse sur les différences inter-sexes pour ce qui a trait à la personnalité et aux comportements subséquents. Lesrésultats obtenus sont en accord avec ceux de Brizendine (2006) qui montrent qu'il n'y a pas de différences notables entre hommes et femmes au plan cognitif, celles-ci n'étant manifestes qu'au niveau des dimensions affective et conative de la personnalité des individus. La perception étant partie intégrante de la cognition, il semble donc normal qu'on ne puisse pas observer de différencessignificatives entre les groupes de sujets examinés. Pour ces auteurs, les différences qui apparaissent au niveau de l'affectivité et des conduites sociales trouvent leur origine dans les pratiques éducatives parentales différenciées selon le sexe de l'individu. Compte tenu du fait que nous contrôlons le niveau d'études en ne prenant en considération que des individus ayant obtenule Brevet d'Etudes du Premier Cycle (BEPC) ou un diplôme équivalent dans le cadre de notre étude, l'effet de la culture inculquée par le milieu familial ousociala probablement été atténué.

Par contre, les résultats que nous avons obtenus diffèrent de ceux de Holloway et al. (1993), Joseph (2000), et Tse et al. (2002). Ces auteurs ont étudié les aspects structuraux et fonctionnels du cerveau de l'homme et de celui de la femme. Ils ont démontré l'existence de différences dans la façon dont le cerveau féminin oumasculin traite les stimuli, entend, voit, sent, évalue ce que les autres éprouvent. Et ces différences observées dans le comportement et les processus mentaux seraient engendréespardes différences anatomiques et physiologiques entre hommes et femmes. Toutefois, il importe de noter que les facteurs biologiques ne constituent que des prédispositions. Ce sont les facteurs psychologiques, en l'occurrence les facteurs de milieu qui actualisent ces potentialités et donc seraient à l'origine des différences de perception intersexuelle. Il est aussi admis avec Huteau (1995) que ces 
prédispositions agissent à des degrés divers sur la structuration du milieu aussi bien physique que relationnel.

Sous le sceau de ce qui précède, il semblerait que les facteurs biologiques et environnementaux interagissent dans la détermination du comportement perceptif des individus à l'égard des personnes porteuses de trisomie 21. Cet aspect des choses pourrait faire l'objet d'études ultérieures pour l'approfondissement des relations entre les variables à l'étude.

\section{Conclusion}

La présente étude s'inscrit dans la perspective des travaux qui se sontintéressés aux différences interindividuelles dans la perception des personnes porteuses de handicap mental. Nous avons émis principalement deux hypothèses. Selon la première hypothèse, les individus qui entretiennent une familiarité de type relationnel avec les personnes trisomiques ont une perception plus positive de la trisomie 21 que leurs pairs entretenant une familiarité de type cognitif avec celles-ci. La seconde hypothèse postule que la proportion de femmes ayant une perception positive de la trisomie 21 est supérieure à celle des hommes. La mise à l'épreuve des faits de ces hypothèses confirme la première, tandis qu'elle infirme la seconde. Cela montre que les hommes et les femmes sont, au même titre, à même de prendre en charge efficacement les enfants porteurs de handicap. De même, ces résultats suggèrent de mettre l'accent sur le contact physique en vue de modifier la perception, voire l'attitude à l'égard de ces enfants, notamment ceux atteints de trisomie 21. Ceci pourrait favoriser leur intégration scolaire, professionnelle et sociale.

\section{References:}

1. Arnold, B. (1960). Emotion and personality. New York: Colombia University Press.

2. Attrobra, N. M. S. (2015). Méthode syllabique, moyen d'initiation $d u$ déficient intellectuel à la lecture, mémoire de fin de cycle, Institut Nationale de Formation Sociale (I.N.F.S), Abidjan 71p. (Non publié).

3. Ayral, S. (2011). La fabrique des garçons, sanctions et genre au collège. Paris: PUF.

4. Baron-Cohen, S. (2003).The essential Difference. The thruth Allport, G. W. (1954). The nature of prejudice.Addison-Wesley: Reading, About the Male and the Female Brain.Jackson: Perseus Books.

5. Bennacer, H. (2005). Le climat de la classe : élaboration d'une échelle adaptée aux collégiens français et prédiction de la performance scolaire. Revue de psychoéducation, 2, 233-263. 
6. Bless, G. (2009). Pédagogie curative différentielle: IntégrationSéparation. Documents de cours non publiés, Institut de Pédagogie Curative, Université de Fribourg, Suisse.

7. Brizendine, L. (2006). Les secrets du cerveau féminin. Paris:Grasset.

8. Bruner, J. S. \& Goodman, G. (1947). Value and Need as Organizing Factors in Perception.f. An. Soc. Psycho, (47) 33-44.

9. Clozel, C-M. (2007). Pourquoi les petits garçons ne sont pas des petites filles... Un secret biengardé. St-Laurent:Triptyque.

10. Cross, H., Halcomb, A., \& Matter, C.G. (1967). Impreting or exposure learning in rats given early auditory stimulation.Psychonomic Science, 7, 233-234.

11. Djider, Z., Murat, F., \& Robin, I. (2003). Motivation et performances : les filles creusent l'écart. Bulletin de l'INSEE, 886, 16-30.

12. Dovidio, J.F., Gaertner, S.L., \& Loux, S. (2000). Subjective experiences and intergroup relations: the role of positive affect. In: $\mathrm{H}$. Bless and J. P. Forgas (ed), The message within. The role of subjective experience in social cognition and behavior (pp.34-71).Philadelphia : PsychologyPress.

13. Duru-Bellat, M. (1994). Filles et garçons à l'école, approche sociologiques et psychosociales. Note de synthèse 2. La construction scolaire des différences entre les sexes, 110, 3-9.

14. Duru-Bellat, M. (1995). Filles et garçons à l'école, approches sociologiques et psycho-sociales. Revue française de pédagogie, $110,75-109$.

15. Eagly, A. H. \& Crowley, M. (1986). Gender and helping behavior. Psychological Bulletin, 100, 283-308.

16. Fournier, M. (2014). Masculin-féminin pluriel. Auxerre : Sciences humaines.

17. Hetebrij, J. \& Racine, G. (2011). Perception du handicap mental par la société. Mémoire d'art en pédagogie curative clinique et éducation spécialisée (non publié).Friboug : Université de Fribourg, Institut de Pédagogie Curative.

18. Huteau, M. (1995). Manuel de psychologie différentielle. Paris : Dunod.

19. Kanga, A. A. B. (2018). Type de famille monoparentale, genre et contrôle de soi chez des élèves du primaire à Abidjan. Thèse de Doctorat de Psychologie (non publié). Abidjan : Université de Félix Houphouët-Boigny.

20. Kimura, D. (2000). Cerveau d'homme, cerveau de femme. Paris : Odile Jacob.

21. Kolb, B. \& Whisaw, I.Q. (2008). Cerveau et comportement. Paris : De Boeck. 
22. Langer, R., Bashner, R. S., \& Chanowlzt, B. (1985). Decreasing prejudice by increasing discrimination.Journal of Personality and Social Psychology, 49, 113-120.

23. Le Maner-Idrissi, G. (1997). L'identité sexuée. Paris: Dunod.

24. LLedo, P. M. \& Vinvent, J. D. (2012). Le cerveau sur mesure. Paris : Odile Jacob.

25. N'douba, B.F. (2011). Réflexion à propos des différences de personnalité inter-sexes. Glotodidactica, Biannual Journal of AppliedLinguistics, 2, 13-27.

26. OMS (2001). Classification internationale $d u$ fonctionnement, $d u$ handicap et de la santé (CIF). Genève.

27. OMS (2008). Classification statistique internationale des maladies et des problèmes de santé connexes. 10ème révision, Genève.

28. Reuchlin, M. (1995). Psychologie. Paris: P.U.F.

29. Reuchlin, M. (1969). La psychologie différentielle. Paris: P.U.F.

30. Rohmer, O. \& Louvet, E. (2000). Les images sociales suscitées par la personne présentant un handicap moteur. La santé de l'Homme, 50, 32-34.

31. Shachter, S. \& Singer, J. E. (1962). Cognitive, social and physiologicaldeterminants of emotional state. Psychologicalreview, 69, 379- 399.

32. Solano, C. (2010). Les trois cerveaux sexuels. Paris : Robert Laffont.

33. Super, D. E., Savikas, M., \& Super, C. (1996). The life-span, life space approach to careers.In D. Brown, L. Brooks and Coll., Career choice and development (3rd ed. pp121-178).San Francisco: Jossey-Bass. 